\title{
Consommation de lait dans les bars laitiers de la ville de N'Djamena au Tchad
}

\author{
M.O. Koussou ${ }^{1 *}$ G. Duteurtre ${ }^{2}$ L.Y. Mopate ${ }^{1}$
}

\begin{abstract}
Mots-clés
Produit laitier - Lait fermenté Consommation - Comportement du consommateur - Zone urbaine Tchad.
\end{abstract}

\begin{abstract}
Résumé
N'Djamena, la capitale du Tchad, connaît depuis dix ans un développement de la consommation hors domicile de lait au sein de petites boutiques appelées les bars laitiers. Pour appréhender les raisons de cet engouement nouveau, une enquête transversale a été réalisée en mars - avril 2007 auprès de 180 consommateurs dans 36 bars laitiers de la capitale. Les consommateurs enquêtés avaient un âge moyen de 31 ans, 54 p. 100 étaient des célibataires, 84 p. 100 étaient originaires de la zone saharo-sahélienne, et la moitié d'entre eux exerçaient dans le secteur informel. Plus d'un tiers des clients (37 p. 100) achetaient les produits pour les emporter, tandis que le reste les consommait sur place. La consommation hors domicile se faisait surtout le soir (79 p. 100). Les produits consommés étaient le lait frais sucré (halib) et le lait fermenté entier (rayeb), souvent accompagnés de pain. Les quantités moyennes consommées par client étaient de $11,5 \mathrm{~L} /$ mois pour le lait frais et de 19,5 L/mois pour le rayeb. Ces quantités variaient selon la saison et l'origine géographique des consommateurs. Le marché connaissait ainsi un pic de consommation en saison sèche chaude, lorsque la production était la plus basse. Cependant, en raison de l'existence de contrats d'approvisionnement des bars laitiers, le prix de ces deux produits était stable pendant toute l'année à $1000 \mathrm{Fcfa} / \mathrm{L}$. Le caractère fermier du lait frais et du lait fermenté entier était déterminant dans le choix de consommation. Des propositions sont formulées pour améliorer l'offre laitière durant la saison chaude afin de satisfaire une demande croissante.
\end{abstract}

1. Laboratoire de recherches vétérinaires et zootechniques, BP 433, N'Djamena, Tchad.

2. Isra-Bame, Dakar, Sénégal ; Cirad, UPR Systèmes d'élevage, Montpellier, France.

* Auteur pour la corespondance

Tél. : +235 6253425 ; e-mail : koussou59@yahoo.fr

\section{INTRODUCTION}

Le lait est l'aliment de base de nombreux peuples d'éleveurs d'Afrique subsaharienne. Le lait et les produits laitiers présentent des qualités nutritionnelles importantes pour les populations des villes africaines ; les protéines du lait complètent en effet utilement les rations alimentaires souvent constituées à partir de céréales et de tubercules (8). En Afrique au sud du Sahara, la consommation des produits laitiers par habitant reste pourtant extrêmement faible : en moyenne $36,4 \mathrm{~kg} / \mathrm{habitant} / \mathrm{an}$, avec de grandes disparités entre les régions, contre 78,5 kg/habitant/an dans le monde (9).

N'Djamena, de par son statut de capitale du Tchad et la densité du flux migratoire qu'elle accueille, est devenue une ville surpeuplée 
et cosmopolite. Elle constitue ainsi le premier centre de consommation des produits laitiers au Tchad dont le marché a été évalué en 1997 à 50000 litres de lait par jour (13). L'accroissement démographique y génère une demande croissante.

Alors que certains auteurs avaient pronostiqué le développement rapide des importations pour répondre à cette croissance du marché, des études de terrain ont révélé que les circuits de commercialisation du lait de brousse étaient particulièrement dynamiques. Ces filières locales impliquent des collecteurs à mobylette et alimentent en lait frais de petites unités de transformation ou bars laitiers situées à l'intérieur de la ville (4). Ces circuits génèrent des débouchés importants pour les producteurs situés en périphérie de la capitale (7).

Deux recensements conduits à quatre ans d'intervalle ont révélé que le nombre de bars laitiers à N'Djamena avait plus que doublé entre 1999 et 2002, passant de 141 à 318 et avec 74 p. 100 d'entre eux qui avaient moins de cinq ans d'existence (4). L'explication tient au développement de la consommation hors domicile du lait entier sucré (halib) et du lait entier fermenté local (rayeb). Ces produits correspondent à des besoins nouveaux des populations urbaines pour des boissons rafraîchissantes. Cependant, aucune étude auprès des consommateurs hors domicile n'avait été menée jusqu'ici.

La présente étude s'est fixée pour objectifs de déterminer le profil des consommateurs, d'analyser leur comportement de consommation au cours de l'année et d'en déduire les conséquences sur la demande en lait. Les résultats pourraient permettre l'élaboration de stratégies d'appui au secteur de la distribution du lait local.

\section{MATERIEL ET METHODES}

Un recensement des bars laitiers actualisé en début d'enquête a conduit à estimer leur nombre entre 360 et 400 . Ils proposaient à leur clientèle du lait frais, du lait fermenté entier ou les deux. Un taux de sondage de 10 p. 100 appliqué à l'effectif total des bars laitiers recensés de la ville a conduit à retenir le nombre de 36 bars laitiers à enquêter. Leur échantillonnage a été effectué proportionnellement à leur concentration par quartier. Au niveau de chaque bar laitier, cinq personnes ont été choisies de manière aléatoire en retenant un client sur trois par ordre d'arrivée. Les entretiens ont eu lieu sur les lieux de consommation entre $16 \mathrm{~h}$ et $21 \mathrm{~h}$ et sur la base d'un questionnaire semi-ouvert. L'année est divisée en trois saisons : la saison sèche chaude (ou saison chaude) qui précède l'arrivée des pluies (mars à mai) où la température moyenne est de $32,3{ }^{\circ} \mathrm{C}$, la saison de pluies (juin à octobre) où la température moyenne mensuelle est de $28,1^{\circ} \mathrm{C}$, et la saison sèche fraîche (novembre à février) avec une moyenne quotidienne de $25,5^{\circ} \mathrm{C}$. L'enquête a été réalisée pendant la saison chaude qui correspondait au pic de fréquentation des bars laitiers (4).

Au total, 180 consommateurs de lait ont été interrogés sur leur profil (âge, sexe, profession, groupe ethnique, origine géographique) et sur leur comportement de consommation (type de produits, fréquences de consommation, quantités consommées, déterminants de la consommation) à la fois pendant la saison chaude (en cours) mais aussi pendant les autres saisons. Les quantités consommées au moment de l'entretien ont été mesurées à l'aide d'un bécher gradué de capacité d'un litre. Celles correspondant aux autres saisons de l'année ont été enregistrées sur la base des déclarations des consommateurs. Le calcul des fréquences de consommation a été effectué avec Excel sous la forme de tableau croisé dynamique. Les moyennes ont été comparées par l'analyse des variances (Anova) avec le logiciel Spss 12.0 pour Windows.

\section{RESULTATS}

\section{Profil des consommateurs}

\section{Selon le genre, l'âge et le statut matrimonial}

La très grande majorité des consommateurs étaient des hommes (87 p. 100 de l'échantillon). Les célibataires ont constitué plus de la moitié de la clientèle des bars laitiers (54 p. 100). L'âge moyen tous sexes confondus était de $31 \pm 8$ ans. Près de 37 p. 100 des célibataires et 58 p. 100 des personnes mariées avaient moins de 30 ans.

\section{Selon la catégorie socioprofessionnelle}

Les consommateurs enquêtés étaient de professions diverses. Les personnes travaillant dans le secteur informel étaient les plus représentées (49 p. 100), suivies des salariés du privé (16 p. 100), des commerçants (16 p. 100) et des salariés du public (11 p. 100) (figure 1). Les travailleurs du secteur informel étaient majoritairement des tâcherons (62 p. 100), des artisans (23 p. 100) et des vendeurs ambulants (15 p. 100). Parmi ces travailleurs du secteur informel, 32 p. 100 étaient des saisonniers.

\section{Selon l'appartenance ethnique et l'origine géographique}

Les consommateurs enquêtés constituaient un ensemble très hétérogène du point de vue ethnique, même si les groupes originaires de la partie centre et nord du pays étaient les plus nombreux (plus de 84 p. 100). Les Arabes (18 p. 100) et les Toubous (17 p. 100) étaient les groupes les plus importants, suivis des Boulalas-koukas (13 p. 100), des Kanembous (12 p. 100) et des Ouaddaiens (10 p. 100). Les populations de la zone méridionale du pays représentaient seulement 9 p. 100 des consommateurs dont la moitié était issue de la région du Mayo-Kébbi. Les populations relevant des groupes « allogènes » (3 p. 100) étaient constituées pour l'essentiel d'Haoussas. Parmi les consommateurs rencontrés, 73 p. 100 ont déclaré résider de manière permanente à N'Djamena, les 27 p. 100 restant y résidaient de manière saisonnière ou temporaire.

\section{Produits consommés : nature, critères, lieu et prix}

Le lait fermenté entier était le produit le plus consommé (69 p. 100) au moment de l'enquête, dans les bars laitiers qui offraient les deux types de produits. La grande majorité des personnes enquêtées (73 p. 100) consommaient un seul des deux types de produits. Les deux types étaient particulièrement appréciés pour leur caractère fermier. Plus de 80 p. 100 des interviewés ont mis en avant l'image positive du lait naturel, c'est-à-dire n'ayant pas été mélangé à d'autres produits ou n'ayant pas fait l'objet d'une fabrication

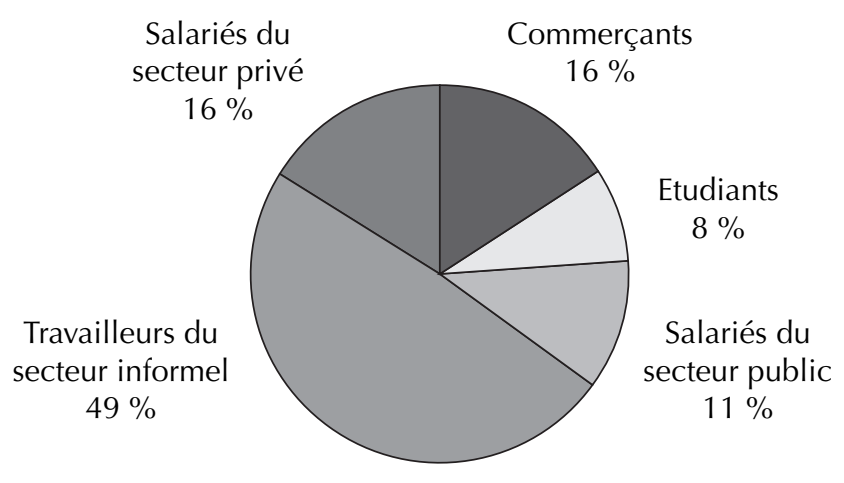

Figure 1 : répartition des consommateurs interrogés selon la catégorie professionnelle. 
par des techniques modernes, qualité soulignée par les enseignes « lait pur de vache » placées sur la devanture de la plupart des bars laitiers.

La majorité des personnes enquêtées (63 p. 100) consommaient les produits sur place, individuellement, contre 37 p. 100 qui les emportaient. Les femmes, dans leur quasi-totalité, emportaient leur commande (22 sur un total de 23).

Les prix et les unités de vente du lait fermenté entier et du lait frais étaient les mêmes : $300 \mathrm{Fcfa}$ pour la grande chope de $0,30 \mathrm{~L}$ et $1000 \mathrm{Fcfa} / \mathrm{L}$. D'autres unités de vente étaient utilisées pour la vente de ces produits laitiers, comme des bouteilles d'eau minérale de 1,5 L ou des bouteilles de Coca-Cola de 0,30 L, pour un prix moyen identique. Les prix de vente du rayeb et du halib ne connaissaient pas de variation saisonnière en dépit de la forte saisonnalité de la production laitière.

\section{Rythmes et horaires de consommation}

La fréquence de consommation quotidienne des produits laitiers dans les bars laitiers a varié selon les saisons. La saison sèche chaude a été la période pendant laquelle le rythme de consommation de deux fois par jour a été le plus courant. En saison pluvieuse, 60 p. 100 des personnes interrogées ont déclaré ne consommer du lait issu des bars laitiers qu'une fois par jour (figure 2).

Près de 12 p. 100 des personnes interrogées ont déclaré ne pas consommer de produits laitiers en saison des pluies et 8 p. 100 en saison froide. Ce groupe de consommateurs fréquentant les bars laitiers uniquement en saison chaude était surtout constitué de travailleurs urbains saisonniers qui regagnaient leur village de juin à décembre pour la saison des cultures.

La fréquence hebdomadaire de consommation a aussi varié selon les saisons. En saison chaude, 80 p. 100 des consommateurs ont déclaré consommer des produits laitiers plus de trois fois par semaine. Un peu plus de la moitié (51 p. 100) en ont consommé deux à trois fois par semaine en saison froide et 61 p. 100 en saison pluvieuse. Pendant ces deux saisons, 10 p. 100 des consommateurs ont consommé du lait une fois par semaine (figure 3).

Le soir, après le coucher du soleil, était le moment de la journée choisi pour la consommation des produits laitiers pour 79 p. 100 des enquêtés. Ce moment de la journée permettait de discuter et de rencontrer amis et parents. Plus des trois quarts des consommateurs ont indiqué leur fidélité à un bar laitier, les raisons évoquées étant la proximité et la qualité des produits (57 p. 100), la proximité uni$150 \mathrm{Fcfa}$ pour la petite chope de $0,15 \mathrm{~L}$, soit un prix moyen de

quement (23 p. 100), le lien de parenté (15 p. 100), les retrouvailles entre amis et la distraction (5 p. 100).

\section{Consommation moyenne mensuelle}

Les quantités moyennes mensuelles consommées ont été de 19,51 L pour le lait fermenté entier et de 11,51 L pour le lait frais, avec une variabilité individuelle importante. En effet, les écartstypes respectifs ont été de 20,88 et 17,03. Les quantités moyennes consommées ont varié de façon significative avec les saisons : elles ont été deux fois plus importantes en saison sèche chaude qu'en saison pluvieuse et froide quel qu'ait été le type de produit. La quantité moyenne de lait frais consommé a été supérieure à celle du lait fermenté, quelle qu'ait été la saison (tableau I).

La consommation journalière a suivi les mêmes variations saisonnières avec une forte proportion d'individus consommant moins d'un demi-litre par jour : 71 et 51 p. 100 respectivement pour le lait fermenté entier et le lait frais. Les quantités moyennes de lait fermenté consommées ont varié significativement suivant le lieu de résidence et la région d'origine des consommateurs. Les clients résidant de manière permanente à N'Djamena consommaient plus que les clients présents à N'Djamena de manière temporaire ou saisonnière, et ceux originaires du Sud consommaient de plus grandes quantités de lait fermenté que ceux originaires du Centre Nord. Le lait frais était lui aussi consommé en plus grandes quantités par les clients résidant en permanence à N'Djamena que par les autres. En revanche, l'enquête n'a pas montré de différence significative entre les quantités moyennes consommées par les clients originaires du Sud et celles consommées par les clients originaires du Centre et du Nord (tableau II).

\section{Tableau I}

Consommation moyenne mensuelle de produits laitiers en fonction de la saison

\begin{tabular}{lrrrr|}
$\begin{array}{l}\text { Type de } \\
\text { produit }\end{array}$ & $\begin{array}{c}\text { Saison } \\
\text { fraîche }\end{array}$ & $\begin{array}{c}\text { Saison } \\
\text { chaude }\end{array}$ & $\begin{array}{c}\text { Saison } \\
\text { pluvieuse }\end{array}$ & ESM \\
\hline Halib (L) & $11,90^{\mathrm{a}}$ & $32,41^{\mathrm{b}}$ & $14,21^{\mathrm{a}}$ & 1,93 \\
Rayeb (L) & $5,78^{\mathrm{a}}$ & $19,95^{\mathrm{b}}$ & $8,83^{\mathrm{a}}$ & 0,98 \\
\hline
\end{tabular}

Les valeurs d'une même ligne suivies d'une lettre différente sont significativement différentes $(\mathrm{P}<0,001)$

ESM : erreur standard de la moyenne

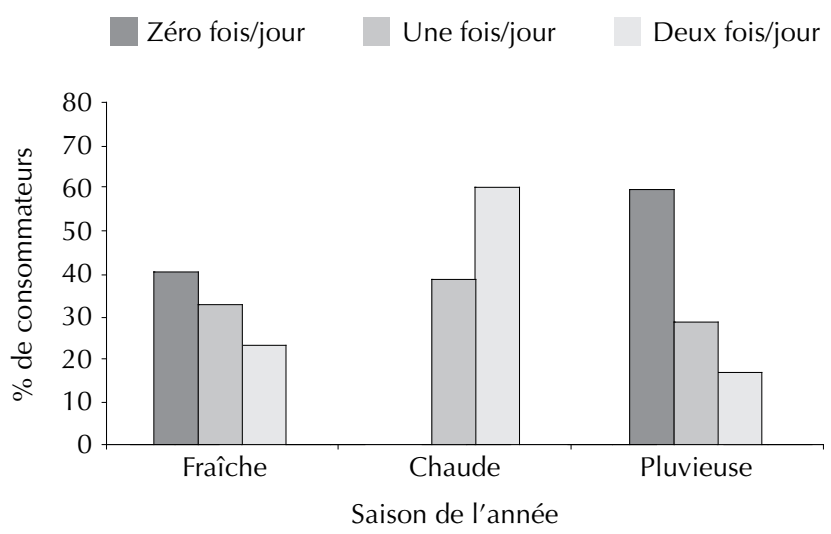

Figure 2 : variation saisonnière de la fréquence journalière de consommation de produits laitiers.
Saison chaude Saison pluvieuse

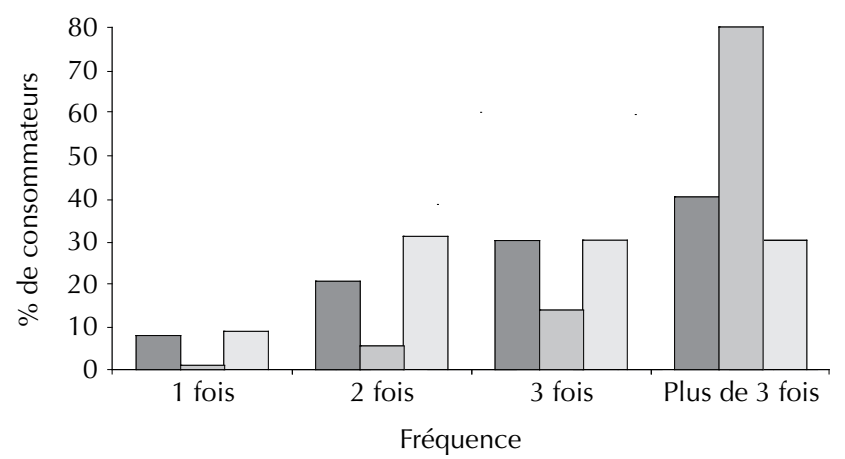

Figure 3 : fréquence hebdomadaire de consommation selon les saisons.
Saison fraîche 


\section{Tableau II}

Consommation moyenne mensuelle de rayeb et de halib selon le lieu de résidence et la région d'origine

\begin{tabular}{llrl} 
Variable & Modalité & Lait fermenté (L) & Lait frais $(\mathbf{L})$ \\
\hline Mobilité & Résident & $13,81^{\mathrm{A}} \pm 17,56$ & $22,14 \pm 21,83$ \\
& Non-résident & $5,89^{\mathrm{B}} \pm 14,26$ & $09,31 \pm 12,48$ \\
$\begin{array}{l}\text { Région } \\
\text { d'origine }\end{array}$ & $\begin{array}{l}\text { Saharo- } \\
\text { sahélienne }\end{array}$ & $10,57^{\mathrm{a}} \pm 16,56$ & $19,03 \pm 20,28$ \\
& $\begin{array}{l}\text { Soudanienne } \\
16,47^{\mathrm{b}} \pm 18,73\end{array}$ & $19,86 \pm 19,24$
\end{tabular}

Les valeurs d'une même colonne suivies d'une lettre différente a, b ou A, B sont significativement différentes respectivement pour $\mathrm{P}<0,05$ et $\mathrm{P}<0,01$

Les produits laitiers étaient consommés avec du pain (dans 67 p. 100 des cas), des gâteaux (3 p. 100) ou sans accompagnement (30 p. 100). Le pain était surtout consommé avec le lait frais. Le rayeb était bu accompagné de pain par seulement la moitié (48 p. 100) des consommateurs.

\section{DISCUSSION}

La consommation de lait hors domicile est une caractéristique de l'urbanité. L'apparition des bars laitiers à N'Djamena est récente. Les résultats des enquêtes menées en 2002, qui avaient conclu à une forte croissance de ce secteur (4), ont été confirmés. Sous l'effet de la demande, le nombre de bars laitiers à N'Djamena a presque doublé entre 1999 et 2002 (cf. plus haut), et est passé à près de 400 en 2007. Cet essor reposait sur le dynamisme des collecteurs laitiers qui, en plus de réaliser le transport et la distribution du produit, proposaient aussi aux boutiques potentiellement intéressées des contrats de livraison « à l'essai », et contribuaient ainsi à l'extension du marché du lait local (4).

Les produits laitiers proposés par les bars laitiers étaient les mêmes qu'en 2002 : le lait frais et le lait fermenté entier. Ces deux produits correspondaient à des besoins nouveaux des populations urbaines pour les boissons rafraîchissantes. Le lait fermenté entier était le produit le plus consommé. Son importance dans les habitudes de consommation urbaines de produits laitiers a été rapportée par d'autres auteurs $(1,9)$.

La clientèle des bars laitiers au moment de l'enquête était dans l'ensemble jeune, masculine et constituée pour plus de la moitié de célibataires. A N'Djamena, les jeunes de moins de 30 ans représentaient 76,4 p. 100 de la population (11). Certains de ces jeunes qui exerçaient des petits métiers n'avaient ni le temps, ni les moyens de préparer des repas à domicile. Ils avaient alors recours à la restauration hors domicile. La consommation de lait avec du pain tenait lieu de repas du soir. Le taux d'accroissement annuel de la population de la ville $(8,4$ p. $100 ; 10)$ est l'un des plus élevé en Afrique subsaharienne et laisse entrevoir une augmentation rapide de la demande urbaine en lait.

Les femmes étaient très peu représentées parmi les consommateurs, alors qu'elles constituaient 52 p. 100 de la population de N'Djamena (10), et les seules clientes rencontrées pratiquaient l'achat « à emporter ». Cette défection des bars laitiers par les femmes pouvait s'expliquer par les conventions et habitudes culturelles et religieuses.
Si les habitants du nord et du centre du pays, consommateurs traditionnels de lait, représentaient la grande majorité des clients des bars laitiers, un nombre non négligeable était originaire du sud. La ville, lieu de brassage de populations diverses, est aussi le lieu de diffusion de nouvelles habitudes de consommation de lait, notamment chez les jeunes originaires de la zone soudanienne qui n'en étaient pas traditionnellement consommateurs, à l'exception des agropasteurs moundang, toupouri ou massa. Le regain d'intérêt des habitants de la zone méridionale pour les produits laitiers a été aussi observé dans la ville de Moundou située en pleine savane au sud du pays (3).

La saison a conditionné le niveau de la demande en lait et produits laitiers : plus faible en saison des pluies et en saison fraîche, la consommation de lait a nettement augmenté en saison sèche et chaude. Le lait frais et le lait fermenté entier possèdent un réel pouvoir rafraîchissant et réhydratant et sont ainsi consommés par un plus grand nombre de personnes (9) en période de fortes chaleurs. C'est le cas à N'Djamena où, de mars à juin, la température moyenne journalière dépasse souvent $30^{\circ} \mathrm{C}$. Paradoxalement, c'est pendant cette période que la production laitière est à son plus bas niveau. Les entretiens avec les responsables des bars laitiers effectués au cours des enquêtes ont d'ailleurs souligné que la saison sèche chaude représentait une saison critique pour l'approvisionnement des bars laitiers.

Cependant, en dépit d'une forte saisonnalité de la production, les prix de vente du lait frais et du lait fermenté entier ne connaissent pas de variations saisonnières, à cause, d'une part, de la forte demande de ces produits tout au long de l'année $(4,6)$ et, d'autre part, du risque lié à la périssabilité du produit, qui implique l'établissement de contrats d'approvisionnement. Les contrats avec des «quarantiers » (livreurs à mobylette) concernent 93 p. 100 des commerces et sont basés sur des accords sur les quantités et les prix de vente (4).

Metzger et coll. (9) rapportent qu'un des obstacles majeurs à la consommation régulière de lait et de produits laitiers en Afrique est la faiblesse du pouvoir d'achat ou, autrement dit, un prix trop élevé du lait et des produits laitiers. A N'Djamena, le prix n'a pas semblé être un obstacle à la consommation individuelle et hors domicile des produits laitiers locaux. C'étaient plutôt les facteurs socioculturels qui étaient déterminants dans le comportement de consommation de produits laitiers hors domicile dans la capitale. Toutefois, on peut anticiper qu'une baisse du prix du lait résultant d'une augmentation de l'offre pourrait conduire à l'augmentation des quantités consommées.

Des efforts seraient nécessaires pour favoriser l'adéquation entre l'offre et la demande, c'est-à-dire en particulier pour augmenter l'offre en saison sèche chaude. L'élargissement de la zone de collecte a été jusque-là la principale réponse à l'augmentation de la demande (7). Des techniques simples, comme la stabulation (2) et la complémentation des vaches (12) appliquées dans des contextes similaires permettaient le maintien et l'amélioration de la production laitière en saison sèche et la génération de revenus pour les familles d'éleveurs. L'utilisation plus systématique des sous-produits agro-industriels par les éleveurs pour complémenter leurs animaux pourrait permettre une meilleure adéquation de l'offre et de la demande. Des essais d'améliorations génétiques par croisement des races locales avec des races plus performantes sont aussi envisageables autour des centres urbains où la demande est forte.

Malgré leur succès, les produits laitiers locaux commercialisés dans les bars laitiers ne présentent pas de garantie formelle sur le plan sanitaire pour les consommateurs. Des études sur la qualité de ces produits semblent nécessaires pour évaluer les risques de santé publique liés à la consommation de lait dans les bars laitiers. Par 
ailleurs, pour améliorer la qualité de lait commercialisé, l'accent doit être mis sur la sensibilisation et la formation des acteurs de la filière aux pratiques d'hygiène du lait. Les bars laitiers pourraient d'ailleurs avoir un impact plus important sur les pratiques d'élevage si elles renforçaient leurs liens contractuels en amont. La mise en place d'un label « lait pur de vache » appuyé par des structures de développement (projet ou services de l'élevage) pourraient permettre la mise en place de ces « bonnes pratiques » (4).

Par ailleurs, l'essor des bars laitiers se heurte à un contexte institutionnel défavorable qui concerne notamment le déficit en électricité et l'absence de cadre de concertation pour orienter les politiques et les projets vers une meilleure prise en compte des besoins des micro- et des petites entreprises laitières (4).

Si de nombreux pays sahéliens ont recours à la poudre de lait importée pour faire face à l'augmentation de la demande urbaine, l'importation de la poudre de lait n'est pas le seul recours. Dans le cas du Tchad, on constate un regain d'intérêt pour les produits laitiers locaux qui s'illustre par le développement des bars laitiers. Au total, les produits laitiers locaux représentent jusqu'à 65 p. 100 des produits laitiers commercialisés au Tchad $(5,13)$.

\section{- CONCLUSION}

La consommation des produits laitiers hors domicile est en plein essor en raison d'une forte croissance démographique et d'une demande spécifique pour des produits traditionnels à base de lait local : le halib et le rayeb. Les bars laitiers, qui constituent des micro-entreprises laitières, apparaissent comme les principaux moteurs du développement de la filière locale. Les clients de ces micro-entreprises sont essentiellement des hommes jeunes, originaires du nord et du centre, pour qui la restauration hors domicile constitue une solution pour les repas du soir. Ils consomment le halib et le rayeb en boisson généralement accompagnée de pain. Cette consommation est répartie sur toute l'année, mais est plus importante en saison chaude. Ce pic de la consommation correspond à une période de faible production mais ne se traduit pas par une hausse des prix, car la quasi-totalité de l'approvisionnement des bars laitiers est assurée grâce des contrats de livraisons pour lesquels les quantités et les prix sont fixés à l'avance. Ces nouvelles habitudes alimentaires se diffusent au sein des populations de la capitale pour lesquelles le « lait pur de vache » semble constituer à la fois un aliment, un lien social et une part d'identité.

\section{Remerciements}

La présente étude a été réalisée grâce à un appui financier du Projet d'appui aux services agricoles et aux organisations des producteurs (Psaop). Nous exprimons nos remerciements à la coordination dudit projet, aux collecteurs de lait et aux unités de transformation pour leur collaboration.

\section{BIBLIOGRAPHIE}

1. DIEYE P.N., BROUTIN C., BA DIAO M., DUTEURTRE G., LY C., 2005. Synthèse bibliographique sur les filières laitières au Sénégal. Document de travail n 1. Dakar, Sénégal, Repol, 47 p.

2. DIEYE N.P, FAYE A, SEYDI M. CISSE S.A., 2002. Production laitière périurbaine et amélioration des revenus des petits producteurs. Cah. Agric., $11: 251-257$.

3. DUTEURTRE G, ATTEYEH A, 2000. Le lait à Moundou, témoin de l'intégration marchande des élevages pastoraux au sud du Tchad. Revue Elev. Méd. vét. Pays trop., 53 : 299-306.

4. DUTEURTRE G., KOUSSOU M.O., SOULEYMANE N'G., 2005. Les «bars laitiers " de N'Djamena (Tchad). Des petites entreprises qui valorisent le lait de brousse. In : Raimond C., Garine E., Langlois O. éds, Ressources vivrières et choix alimentaires dans le bassin du lac Tchad, Actes $\mathrm{XI}^{\mathrm{e}}$ Coll. Méga-Tchad, Université de Paris X, Nanterre, France, 20-22 nov. 2002. Paris, France, IRD, p. 435-456.

5. KOUSSOU M.O. 2001. L'approvisionnement de la ville de N’Djamena en produits laitiers. In : Duteurtre G., Meyer C. éds, Marchés urbains et développement laitier en Afrique subsaharienne. Montpellier, France, Cirad, p. 75-80.

6. KOUSSOU M.O., 2004. Dynamique des innovations dans le secteur de l'élevage au Tchad : le cas de la filière d'approvisionnement de la ville de N'Djamena en lait. Mémoire DEA "Emts », INA-PG, Paris, France, $44 \mathrm{p}$

7. KOUSSOU M.O., MOPATE Y.L., 2006. Le basin d'approvisionnement de la ville de $\mathrm{N}^{\prime} \mathrm{Dj}$ jamena en lait : zonage et typologie des élevages laitiers. Revue afr. Santé Prod. Anim., 4 : 39-44.

8. MATHERON G., 2001. Discours d'ouverture. In : Duteurtre G. Meyer C. éds, Marchés urbains et développement laitier en Afrique subsaharienne. Montpellier, France, Cirad, p. 13-15.

9. METZGER R., CENTRES J.M., THOMAS L., LAMBERT J.C., 1995. L'approvisionnement des villes africaines en lait et produits laitiers. Rome, Italie, FAO, 102 p. (Production et santé animale, $n^{\circ} 124$ )

10. MINISTERE DE L'ECONOMIE ET DU PLAN, 1995. Recensement général de la population et de l'habitat en 1993, vol. 3 Analyse, tome 2 Etat de la population. N'Djamena, Tchad, ministère de l'Economie et du Plan / BCR, $164 \mathrm{p}$.

11. MINISTERE DES FINANCES, DE L'ECONOMIE ET DU PLAN, 2006. Tchad, profil de pauvreté. II enquête sur la consommation et le secteur informel. N'Djamena, Tchad, ministère des Finances, de I'Economie et du Plan / Inseed, $124 \mathrm{p}$.

12. NJOYA A., LOKO DICKA B., 1997. Production laitière périurbaine des bovins au Nord-Cameroun. In : Seiny Boukar L., Poulain J.F. Faure G. éds, Actes atelier Agricultures des savanes du NordCameroun, Garoua, Cameroun, 25-29 nov. 1996, p. 515-516.

13. ZAFINDRAJAONA P.S., GAUTHIER J., BOUCHEL D., 1997. Mission d'identification d'un projet visant à I'amélioration de I'approvisionnement laitier de N'Djamena. Montpellier, France, Cirad-emvt, 78 p. (Rapport $n^{\circ} 97.022$ )

Accepté le 21.10.2009 


\section{Summary}

Koussou M.O., Duteurtre G., Mopate L.Y. Milk Consumption in the Milk Bars of the City of N'Djamena in Chad

Out-of-home milk consumption has been increasing for the past ten years in N'Djamena, capital of Chad, through small shops called milk bars. In order to understand the reasons for this new dynamic, 180 consumers chosen at random in 36 of these places were interviewed by means of a transversal survey conducted in March-April 2007. The surveyed consumers were on average 31 years old, unmarried for the majority $(54 \%)$, originated from the Sahara-Sahelian region $(84 \%)$ and worked in the informal sector (half of them). More than one third (37\%) bought milk "to take away" and the rest consumed it in the bars, mostly in the evening $(79 \%)$. The dairy products consisted in sweetened fresh milk (halib) and cultured whole milk (rayeb) often consumed with bread. Average consumed quantities per client were $11.5 \mathrm{~L} /$ month for fresh milk and $19.5 \mathrm{~L} /$ month for rayeb. Quantities varied according to the season and the origin of the client. A consumption peak occurred during the hot dry season when production was at its lowest level. However, the price of these two products was stable at 1000 FCFA/L throughout the year, because of the existence of supply contracts with the milk bars. The farm origin of fresh or fermented whole milk greatly influenced the consumers' choice. Suggestions are made to improve milk supply during the hot season so as to meet the increasing demand.

Keywords: Milk product - Cultured milk - Consumption Consumer behavior - Urban area - Chad.

\section{Resumen}

Koussou M.O., Duteurtre G., Mopate L.Y. Consumo de leche en los bares lecheros de la ciudad de N'Djamena en Chad

N'Djamena, capital de Chad, goza desde hace diez años de un desarrollo del consumo de leche fuera del domicilio, en el seno de pequeñas boutiques Ilamadas bares lecheros. Para comprender las razones de esta nueva afición, se llevó a cabo una encuesta transversal entre marzo y abril del 2007, con 180 consumidores en 36 bares lecheros de la capital. Los consumidores interrogados tenían una edad media de 31 años, $54 \%$ de ellos eran solteros, $84 \%$ originarios de la zona saharo-sahelina y la mitad ejercía en el sector informal. Más de un tercio de los clientes (37\%) compraban los productos para llevar, mientras que el resto los consumía en el sitio mismo. El consumo fuera del domicilio se daba sobre todo en la noche (79\%). Los productos consumidos fueron leche dulce fresca (halib) y leche agria (o fermentada) entera (rayeb), a menudo acompañados de pan. Las cantidades promedio consumidas por los clientes fue de $11,5 \mathrm{~L} /$ mes de leche fresca y $19,5 \mathrm{~L} /$ mes de rayeb. Estas cantidades variaron según la estación y el origen geográfico de los consumidores. El mercado conoce así un "pico" de consumo durante la estación seca y caliente, cuando la producción de leche es más baja. Sin embargo, debido a la existencia de contratos de abastecimiento de los bares lecheros, el precio de estos dos productos fue estable durante todo el año, a 1000 FCFA/L. El carácter casero de la leche fresca y de la leche fermentada entera fue determinante en la escogencia del consumo. Se formulan propuestas con el fin de mejorar la oferta lechera durante la estación cálida, con el fin de satisfacer una demanda creciente.

Palabras clave: Producto lácteo - Leche fermentada Consumo - Comportamiento del consumidor - Zona urbana Chad. 